\title{
Characterization of a Be(p,xn) Neutron Source for Fission Yields Measurements
}

\author{
A. Mattera, ${ }^{1}$ M. Lantz,${ }^{1}$ S. Pomp,${ }^{1, *}$ V. Rakopoulos,${ }^{1}$ A. Solders,${ }^{1}$ P. Andersson, ${ }^{1}$ \\ A. Hjalmarsson, ${ }^{1}$ J. Valldor-Blücher ${ }^{1}$ A.V. Prokofiev, ${ }^{1}$ E. Passoth, ${ }^{1}$ D. Gorelov ${ }^{2}$ H. Penttilä, ${ }^{2}$ \\ S. Rinta-Antila, ${ }^{2}$ R. Bedogni, ${ }^{3}$ A. Gentile, ${ }^{3}$ D. Bortot,${ }^{3}$ A. Esposito, ${ }^{3}$ M.V. Introini, ${ }^{4}$ and A. Pola ${ }^{4}$ \\ ${ }^{1}$ Uppsala University, Uppsala, Sweden \\ ${ }^{2}$ University of Jyväskylä, Jyväskylä, Finland \\ ${ }^{3}$ INFN-LNF Laboratori Nazionali di Frascati, Frascati (Rome), Italy \\ ${ }^{4}$ Politecnico di Milano, Milano, Italy.
}

\begin{abstract}
We report on measurements performed at The Svedberg Laboratory (TSL) to characterize a proton-neutron converter for independent fission yield studies at the IGISOL-JYFLTRAP facility (Jyväskylä, Finland). A 30-MeV proton beam impinged on a $5 \mathrm{~mm}$ water-cooled Beryllium target. Two independent experimental techniques have been used to measure the neutron spectrum: a Timeof-Flight (TOF) system to estimate the high-energy contribution, and a Bonner Sphere Spectrometer to provide precise results from thermal energies up to $20 \mathrm{MeV}$. An overlap between the energy regions covered by the two systems will permit a cross-check of the results from the different techniques. In this paper, the measurement and the analysis technique will be presented together with some preliminary results.
\end{abstract}

\section{INTRODUCTION}

High-quality measurements of independent fission yields from key actinides are desirable at various neutron energies in view of the possible use of fast neutrons in generation-IV reactors and of innovative cycles for the handling of spent nuclear fuel. At the IGISOLJYFLTRAP facility (Jyväskylä, Finland) a new high intensity MCC30/15 cyclotron with proton beams up to $100 \mu \mathrm{A}$ in current and $30 \mathrm{MeV}$ in energy is now available, and a proton-neutron converter has been designed to provide suitable fields to study neutron induced fission yields of different actinides. The study of the fission products will be performed using the JYFLTRAP apparatus, which has been used for measurements of proton-induced independent fissions yields [1].

Different materials and geometries for the protonneutron converter have been investigated, the preferred design being a $5 \mathrm{~mm}$-thick water-cooled Beryllium target. The target is thinner than the stopping length of $30-\mathrm{MeV}$ protons, allowing them to stop in the subsequent layer of cooling water. In this way, the cooling requirements and the hydrogen build-up in the metal, which could both represent major problems at high currents, are significantly reduced. The neutron yield has been studied with Monte Carlo calculations using MCNPX [2] and FLUKA [3, 4]

\footnotetext{
* Corresponding author: stephan.pomp@physics.uu.se
}

simulation codes $[5,6]$, but given the significant energy dependence of the fission cross-section, a good knowledge of the energy spectrum is required and hence, a direct measurement is desired.

The present work describes measurements performed at The Svedberg Laboratory (TSL), Uppsala (Sweden) to characterize the energy spectrum from a mock-up of the proton-neutron converter.

\section{EXPERIMENTAL SETUP}

The available energy at IGISOL-JYFLTRAP was reproduced by degrading the $37.3-\mathrm{MeV}$ proton beam at the PAULA facility [7] of TSL down to $29.8 \mathrm{MeV}$, by means of a 1.015 mm-thick aluminium slab and a path in air.

The degraded proton beam was shaped by two sets of graphite collimators: a first $10 \times 10-\mathrm{mm}^{2} \mathrm{x}-\mathrm{y}$ collimator was used to reduce the size of the beam and a cylindrical collimator with an opening $15 \mathrm{~mm}$ in diameter, positioned in close proximity of the target assembly, was used to avoid any neutron production in the aluminium target holder.

Two independent experimental techniques were used to measure the neutron spectrum: a Time of Flight (TOF) system was used to estimate the high-energy contribution; and a Bonner Sphere Spectrometer (BSS), to measure the neutron energy from the thermal region up to $20 \mathrm{MeV}$.

The BSS was composed of 13 spheres with radii be- 
tween 2 and 12 inches. Details about this system and the results will be reported elsewhere [8].

The TOF setup consisted of a 3.31 BC-501 liquid scintillator from the NORDBALL array [9] placed at different distances $(1.2,2.0$ and $4.8 \mathrm{~m})$ from the source, at an angle of 10 degrees with respect to the beam direction. The distances were selected to obtain a good energy resolution over the whole measured energy interval (see Section III C) and to minimize the wrap-around effect given by the time structure of the proton beam, that had a period of $44.25 \mathrm{~ns}$. The Pulse Shape Discrimination (PSD) capabilities of the liquid scintillator helped to reduce the gamma background. Calibration of the TOF measurement system was provided with two simpler versions of the neutron source: the same proton beam was made to impact on both a thin $(1 \mathrm{~mm})$ and a full-stop $(6 \mathrm{~mm})$ Beryllium target.

Two different Data AcQuisition (DAQ) systems were used simultaneously during the experiment. In both systems, the trigger was provided by an event above threshold detected in the scintillator and the stop signal was given by the radio-frequency (RF) pulse from the cyclotron; the latter signal is delayed with respect to the time protons hit the target according to $T_{\text {stop }}=T_{0}+\Delta t$, where $T_{0}$ is the instant when protons reach the Be target and $\Delta t$ is the delay. It was possible to reconstruct the delay $\Delta t$ from the peaks corresponding to the gamma flashes produced in objects along the beam-line (Fig. 1). The first analogue system provided online PSD with a dedicated NIM module [9]. The information was stored along with the TOF, digitized by a TDC with a $25 \mathrm{ps}$ sampling time, on an event-by-event basis. The energy threshold was varied between 3 values, that were estimated to correspond to about 2, 3 and $7 \mathrm{MeV}$ neutrons.

The second acquisition system used a Multi Channel Analyser (SP Devices ADQ412 High-speed Digitizer) with which the whole pulse shape of each event was stored for off-line PSD. In this case, the threshold is adjustable off-line. The RF signal was also stored to extract the

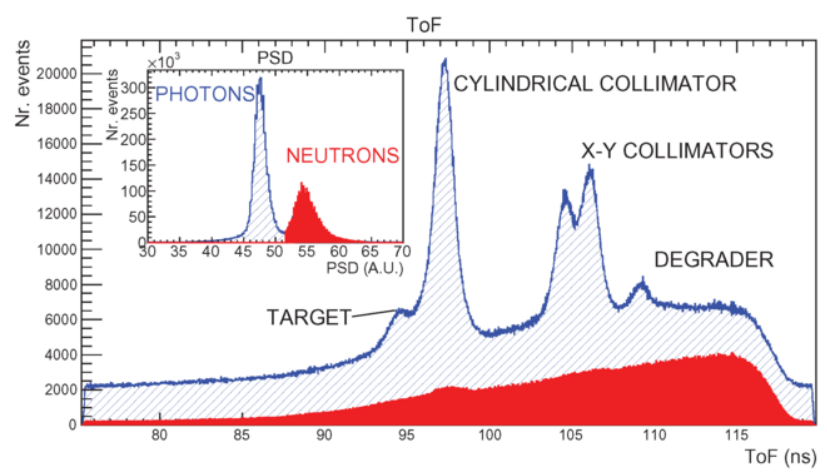

FIG. 1. Raw TOF spectrum. The PSD spectrum in the inset shows the separation between neutrons (solid red) and photons (hatched blue). The peaks in the photon spectrum correspond to production in the beam-line elements. The reduction of the gamma background is evident in the TOF spectrum.
TOF information. The data presented in this paper come from the analogue DAQ: the analysis of the data collected with the Multi Channel Analyser is discussed elsewhere [10].

\section{RESULTS}

\section{A. Measurement of the Background}

The contribution of neutrons not coming from the $\mathrm{Be}(\mathrm{p}, \mathrm{xn})$ source was estimated using the shadow-cones (SC) technique with both the TOF system and the BSS. $50 \mathrm{~cm}$ long shadow-cones $(20 \mathrm{~cm}$ iron $+30 \mathrm{~cm}$ polyethylene) were manufactured in 5 different sizes to accommodate the dimensions of the Bonner spheres and the scintillator and to ensure good shadowing conditions at all distances.

Preliminary Monte Carlo simulations of the neutron transportation in the experimental hall showed that a significant background can be expected: the comparison of a simulated setup with and without SC is shown in Fig. 2. The background mainly originates from low energy neutrons produced in the collimators or other beam-line elements or from neutrons scattered in air and off the walls of the experimental area.

A preliminary result of the TOF measurement is shown in Fig. 3. The threshold cut in the data presented here is such that the contribution from low energy neutrons, that are expected to dominate the background, is suppressed.

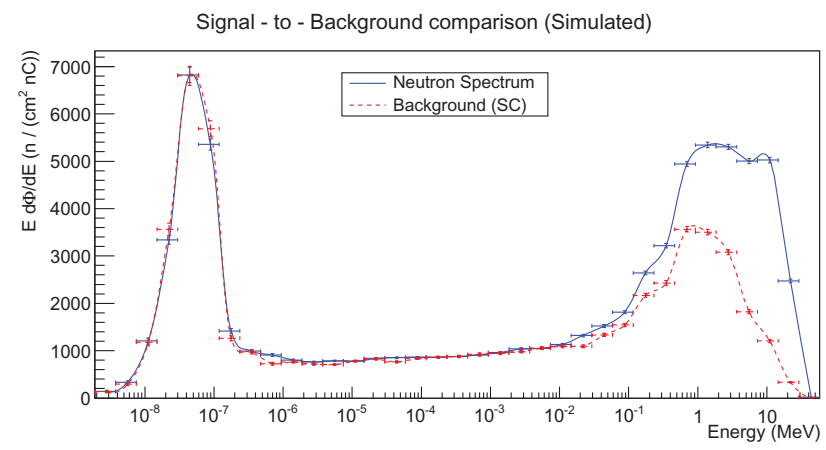

FIG. 2. Comparison of the FLUKA simulated neutron spectrum without (solid blue) and with (dashed red) the shadow cones (SC), to estimate the scattered neutrons background.

\section{B. Pulse Shape Discrimination}

The parameters of the PSD were optimized before the TSL run with a ${ }^{252} \mathrm{Cf} n-\gamma$ source and verified with the neutrons from the Be source. An example of the effect of the PSD is shown in Fig. 1. Despite the very effective reduction of the continuum gamma background and in the gamma peaks at shorter TOFs, it can be noticed that the online PSD has some limitations in subtracting the 


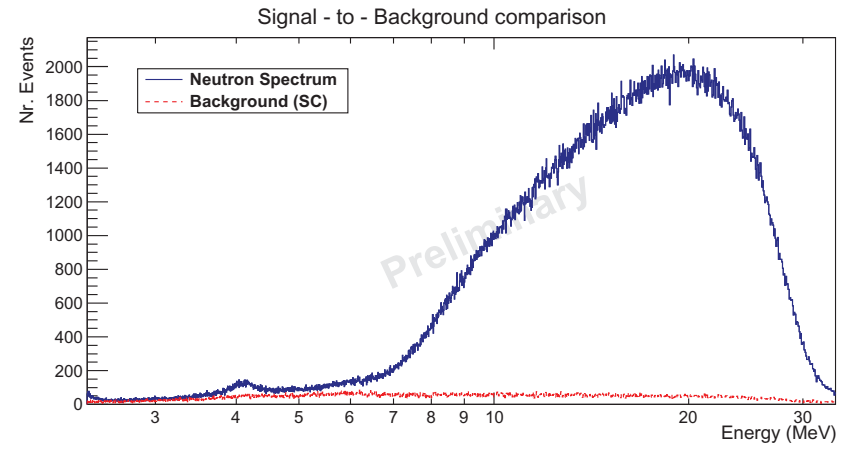

FIG. 3. Comparison of the measured neutron spectrum without (solid blue) and with (dashed red) the shadow cones (SC), to estimate the scattered neutrons background.

intense gamma peak from the collimators and the target itself. This effect could not be explained by simple analysis of the analogue data and was observed for all the combination of distances and thresholds.

\section{Expected Energy Resolution}

In first approximation, the energy resolution $(\delta E / E)$ of the TOF spectrometer depends on the accuracy with which time and distance can be estimated ( $\delta T$ and $\delta L$, respectively), according to

$$
\frac{\delta E}{E}=2\left[\left(\frac{\delta L}{L}\right)^{2}+\left(\frac{\delta T}{T}\right)^{2}\right]^{\frac{1}{2}},
$$

where $L$ is the source-to-detector distance and $T$ is the TOF.

For $20 \mathrm{MeV}$ neutrons, Eq. 1 gives an uncertainty of $4.2 \%$ at $4.8 \mathrm{~m}$, that increases to $17 \%$ at $1.2 \mathrm{~m}$; the uncertainty is dominated by $\delta L$, assumed equal to the half-thickness of the liquid scintillator $\approx 8 \mathrm{~cm}$, whereas $\delta T \approx 1 \mathrm{~ns}$ is dominated by the duration of the proton bunch, that was determined by a dedicated measurement with protons directly impinging on the detector. To improve the energy resolution, the position of interaction of neutrons should be known more accurately, but even in the most favourable scenario $(\delta L \approx 1 \mathrm{~cm})$, the energy resolution cannot be expected to be better than $2.6 \%$ for the farthest distance.

\section{CONCLUSIONS AND FUTURE WORK}

In this paper we presented the experimental setup and the preliminary results of a characterization measurement of the energy spectra of neutrons produced by a $\mathrm{Be}(\mathrm{p}, \mathrm{xn})$ source. The source will be used for neutron-induced fission yield measurements of actinides at the IGISOLJYFLTRAP facility. The measurement used the TOF technique to evaluate the high-energy part of the energy spectrum. The use of a liquid scintillator with neutrongamma discrimination capabilities allowed to reduce the contribution of the gamma background.

The preliminary results presented here were obtained analysing data collected with an analogue DAQ, with online PSD. The limitations of this system are being investigated by analysing the data collected with the MCA, where an off-line pulse shape analysis has been performed event-by-event [10]. In addition to the PSD information and the TOF, the pulse height will help to identify the gamma background.

Monte Carlo simulations are being performed with different computer codes (MCNPX, FLUKA and GEANT4 [11]) to be compared with the measured data. They will also be used to extract the response function of the detector in order to correct the spectra obtained with the detection efficiency at different energies.

Acknowledgements: The work was supported by the European Commission within the Seventh Framework Programme through Fission-2010-ERINDA (project no. 269499), by the Swedish Radiation Safety Authority (SSM), and by the Swedish Nuclear Fuel and Waste Management Co. (SKB).
[1] H. Penttilä et al., Eur. Phys. J. A 48, 43 (2012).

[2] G.W. McKinney et al., Proceedings of the 2006 ANS Winter Meeting (2006).

[3] G. Battistoni et al., Proceedings of the Hadronic Shower Simulation Workshop 2006 896, 31 (2007).

[4] A. Ferrari et al., CERN-2005-10, INFN/TC 05/11, SLAC-R-773 (2005).

[5] A. Solders et al., Nucl. Data Sheets 119, 338 (2014).

[6] M. Lantz et al., submitted to IAEA-F1-TM-42752 (2013).
[7] http://www.tsl.uu.se/irradiation-facilities-tsl/ PAULA-proton-beam-facility/ (Nov. 2013).

[8] R. Bedogni et al., IN PREPARATION.

[9] S.E. Arnell et al., Nucl. Instrum. Methods Phys. Res. A 300, 303 (1991).

[10] V. Rakopoulos et al., accepted in Proceedings of the International Nuclear Physics Conference (2013).

[11] S. Agostinelli, Nucl. Instrum. Methods Phys. Res. A 506, 250 (2003). 